\title{
Study on the realization method of the high-speed train full return air mode
}

\author{
Yifei Zhu, Yugong Xu, Xiangdong Chen \\ School of Mechanical Electronic and Control Engineering, Beijing Jiaotong University, Beijing \\ 100044, China
}

Keywords: high-speed train, full return air mode, carriage, simulation.

\begin{abstract}
With the development of our high speed train, passengers on the train ride comfort requirements are gradually increased. Therefore we should study some emergency cases in car environment. The article explores the use of CFD software method to realize the full return air model, provide the basis for the research of full return air mode so as to the carriage, it has important value to improve the research environment in the carriage.
\end{abstract}

\section{Introduction}

High-speed train carriage airflow directly related to the passengers ride feel, along with the improvement of people's material and cultural level of awareness and self-protection, passenger comfort requirements of increasingly high-speed train [Tanifuji, K, 2008, Meng-ling, 2011, Guo, Y, 2013]. Study of high-speed train carriage environment, the current study focused on general operating conditions of the flow field inside the carriage [Gilbert, T, 2013, Fan, R, 2014, Fan, R., G, 2014,], while the current train encountered a variety of air conditioning and ventilation system in an emergency situation, such as a return air mode few studies, and whether the air conditioning system at this time is still able to meet the requirements of comfort is also unknown [Cao, X, 2013]. In this paper, the use of CFD software for the full realization of the full return air mode to explore, to provide a basis for the study of wind patterns whole back compartment, which is to improve the cabin environment has important significance.

\section{Physical modeling}

High-speed trains in some special cases will be closed ventilation system into the air vents, open the car's full return air mode will soon return and supply air is connected to the car in order to achieve inner loop compartment. Therefore the establishment of the following examples on how to achieve a full return air in the calculation of this function, the model shown in Fig.1.

As can be seen from the figure, the model is a simple two-dimensional model of the carriage, the carriage dimensions of length 20m, height 3m; air inlet on the roof, Return air inlet in the left; with a U-shaped tube connecting the inlet and return air, used to simulate the full return air mode.

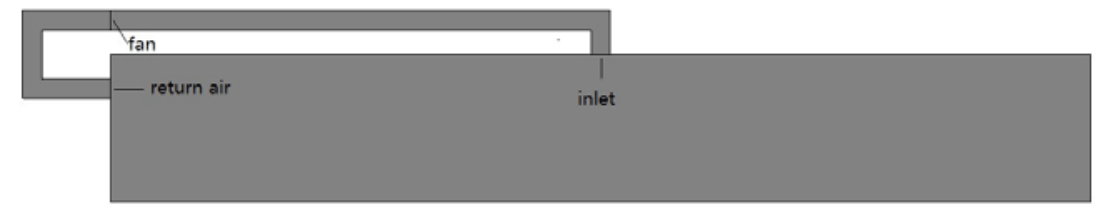

Fig.1 Physical model

\section{Boundary conditions}

Fan boundary conditions: a fan is considered to be infinitely thin, and the discontinuous pressure rise across it is specified as a function of the velocity through the fan. The relationship may be a constant, a polynomial, piecewise-linear, or piecewise-polynomial function, or a user-defined function. In the case of a polynomial, the relationship is of the form. 


$$
\Delta \mathrm{p}=\sum_{n=1}^{N} f_{n} V^{n-1}
$$

Where $\Delta \mathrm{p}$ is the pressure jump, $f_{n}$ are the pressure-jump polynomial coefficients, and $v$ is the magnitude of the local fluid velocity normal to the fan.

Porous Jump Boundary Conditions: porous jump conditions are used to model a thin "membrane" that has known velocity (pressure-drop) characteristics. It is essentially a 1D simplification of the porous media model available for cell zones. Examples of uses for the porous jump condition include modeling pressure drops through screens and filters, and modeling radiators when you are not concerned with heat transfer. This simpler model should be used whenever possible (instead of the full porous media model) because it is more robust and yields better convergence. The thin porous medium has a finite thickness over which the pressure change is defined as a combination of Darcy's Law and an additional inertial loss term:

$$
\Delta \mathrm{p}=-\left(\frac{\mu}{\alpha} v+C_{2} \frac{1}{2} \rho v^{2}\right) \Delta m
$$

Where $\mu$ is the laminar fluid viscosity, $\alpha$ is the permeability of the medium, $C_{2}$ is the pressure-jump coefficient, $v$ is the velocity normal to the porous face, and $\Delta m$ is the thickness of the medium.

While in the U-tube in a position (in the example is arbitrarily defined) is defined at a boundary to simulate the fan, to drive back to the wind as a power source; set cabin wall and a duct wall are no-slip wall boundary, the boundary fan pressure drop 100pa.

\section{Analysis of calculation}

As can be seen from Fig.2, the fan runs produced a driving force, in front of the fan to generate a negative pressure, which will make the air inside of the exhaust port flow play a role in the return air, and in the rear side of the fan generated promote positive pressure air to the cabin air transport and thus play a role.

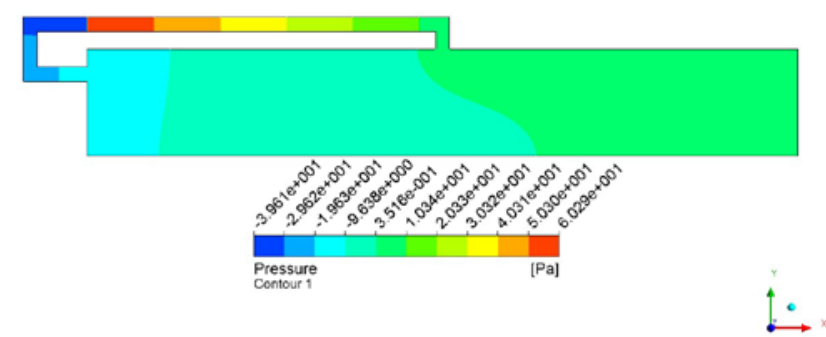

Fig.2 Overall pressure cloud

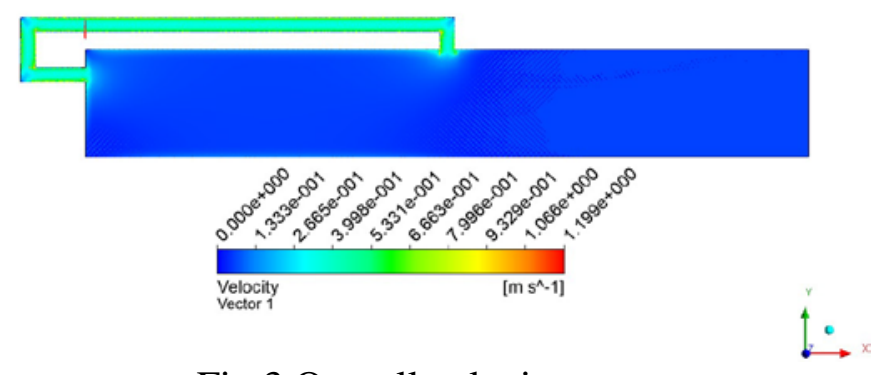

Fig.3 Overall velocity vector

Fig. 3 is the overall speed of the car vector, because the fan to the pressure difference is small, so the speed of the flow field inside a relatively small place only in the return air inlet velocity is slightly larger, and we only focus on return air inlet flow field characteristics, and therefore of the flow field inside the U-shaped tube downplayed. 


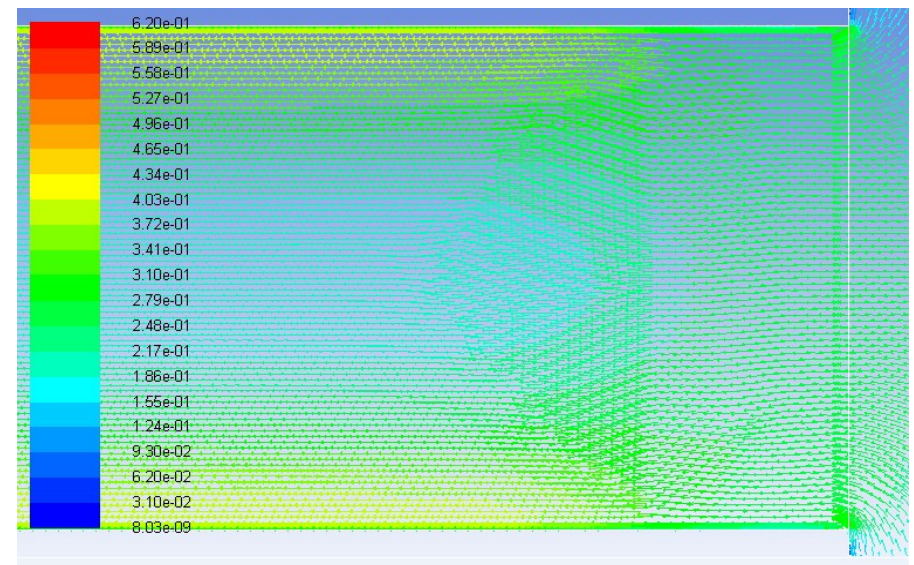

Fig.4 Return air inlet velocity vector

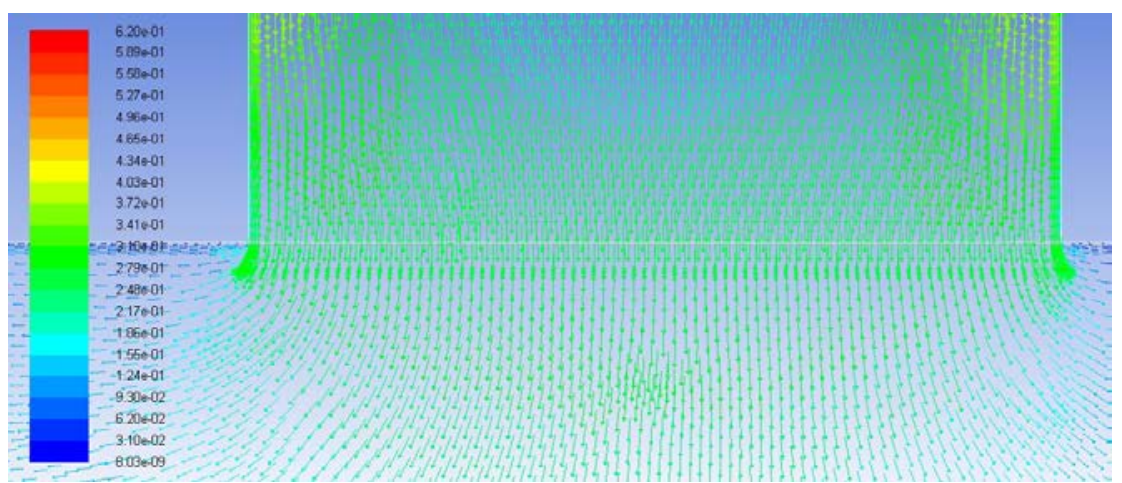

Fig.5 Inlet velocity vector

Fig. 4 and Fig.5 for the return air inlet porous media model was added into the return air velocity vector, velocity can be seen from the figure is more evenly distributed.

Extraction of return air inlet velocity data at the graph shown in Fig.6 and Fig.7, it can be concluded as porous media model after loading inlet and outlet velocity distribution is more uniform.

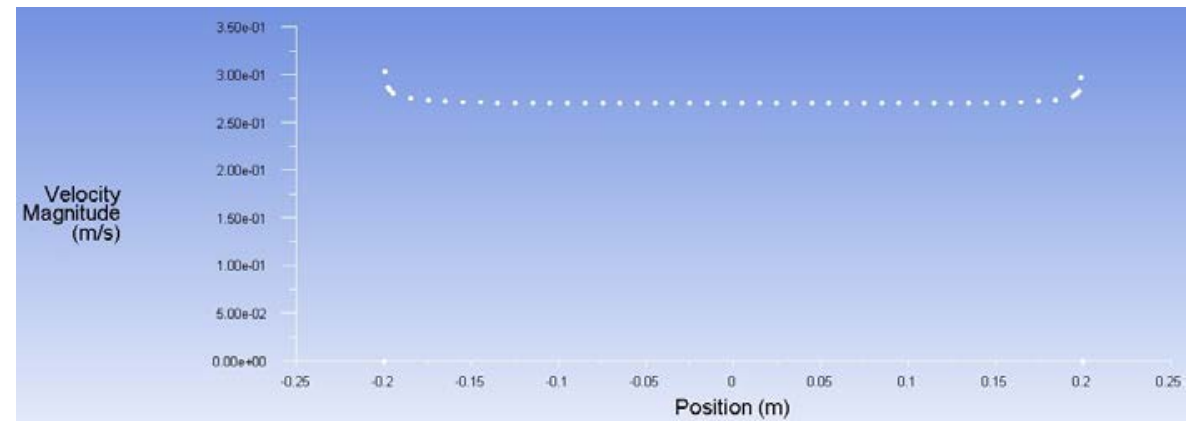

Fig.6 Return air inlet velocity distribution curve

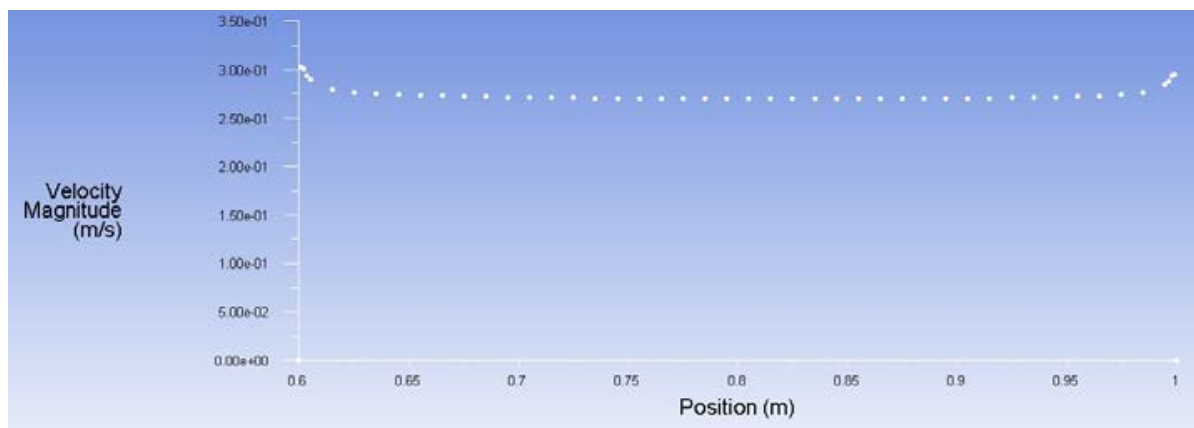

Fig.7 Inlet velocity distribution curve 


\section{Conclusion}

This technology applied to the actual route train compartment model with a boundary as the blower fan drive back to the wind power source in the form of realization; then porous media model to ensure that return air inlet velocity uniformity; and can be up or down a small fan pressure to increase or decrease the flow rate, i.e., for traffic to meet the design value. At this point the return air mode calculation method to determine wind patterns.

\section{Acknowledgment}

This work is supported by grants from the national 863 project (No.2011AA11A103), and the Fundamental Research Funds for the Central Universities (No.2012JBM093). The authors are grateful for this financial support.

\section{References}

[1] Cao, X., et al., Numerical simulation and optimization for the air conditioning and ventilation system in the compartment of urban rail train. China Railway Science, 2013, 34(6): p. 105-109.

[2] Fan, R., et al., Application of sound intensity and partial coherence to identify interior noise sources on the high speed train. Mechanical Systems and Signal Processing, 2014, 46(2): p. 481-493.

[3] Fan, R., G. Meng and Z. Su, The experimental study of the effect of air conditioning system on internal noise within high speed train. International Journal of Vehicle Noise and Vibration, 2014, 10(1-2): p. 93-107.

[4] Guo, Y. and G. Song, Dynamic comfort evaluation of high-speed train based on annoyance rate model. Journal of Northeastern University, 2013, 34(11): p. 1620-1624.

[5] Gilbert, T., C.J. Baker and A. Quinn, Gusts caused by high-speed trains in confined spaces and tunnels. Journal of Wind Engineering and Industrial Aerodynamics, 2013, 121: p. 39-48.

[6] Meng-ling, W., et al., Influence of aerodynamic braking on the pressure wave of a crossing high-speed train. Journal of Zhejiang University Science A, 2011, 12(12): p. 979-84.

[7] Tanifuji, K., K. Sakanoue and S. Kikko, Modelling of aerodynamic force acting on high speed train in tunnel and a measure to improve the riding comfort utilising restriction between cars. Vehicle System Dynamics, 2008, 46(suppl1): p. 1065-75. 